\title{
Surface mapping of carrier density in a GaN wafer using a frequency-agile $\mathrm{THz}$ source
}

\author{
$\underset{\text { sohno@iken.jp }}{\text { Seigo Ohno }}$
}

Akihide Hamano

Katsuhiko Miyamoto

Chisato Suzuki

Hiromasa Ito
RIKEN Sendai, Sendai, Japan

Materials Research Laboratory, Furukawa Co., Ltd., Tsukuba, Japan

RIKEN Sendai, Sendai, Japan

Materials Research Laboratory, Furukawa Co., Ltd., Tsukuba, Japan

RIKEN Sendai, Sendai, Japan

Graduate School of Engineering, Tohoku University

We developed a method for mapping the carrier density on a semiconductor substrate surface based on terahertz (THz)-reflective measurement. Reflectivity in the THz-frequency region away from the optical phonon frequency is sensitive to the carrier density in semiconductors. However, reflectivity in the optical phonon frequency regions is around 1.0, independent of the carrier density. We developed a THz-reflective spectral imaging system using a frequency-agile, ultra-widely tunable $\mathrm{THz}$ source (1-40 THz). Different reflective images were obtained from GaN samples of carrier density $2.5 \times 10^{16} \mathrm{~cm}^{-3}, 1.0 \times 10^{18} \mathrm{~cm}^{-3}$ and $1.5 \times 10^{18} \mathrm{~cm}^{-3}$ using 22.7 and 26.5 THz. The image contrast reflected the GaN crystals' carrier density. [DOI: 10.2971/jeos.2009.09012]

Keywords: imaging systems, terahertz spectroscopy, GaN, carrier density

\section{INTRODUCTION}

Monitoring the carrier density in semiconductors is a practical way of analyzing physical phenomena in these materials. Hall measurements using a four-terminal method are traditionally applied to obtain information on carrier density, mobility, and conductivity in semiconductors. However, measuring the density distribution in a surface of samples is difficult because electrical contact is required. In addition, the destructive nature of this method makes it unsuitable for industrial semiconductor manufacturing. Some studies have applied a nondestructive optical method to measure the surface carrier distribution [1]-[3]. Electromagnetic waves of terahertz (THz)band frequencies are well known to be sensitive to carrier density and optical phonon properties in semiconductors. Huber et al. recently reported extremely high sensitivity and spatial resolution for carriers in a semiconductor using a THz-wave and atomic-force microscopic technique [4]. The sensitivity of THz-waves to carriers enables the near-field nanoscopy using $\mathrm{THz}$-waves to achieve extremely high sensitivity for carriers in a single nanodevice.

Our research group has developed monochromatic ultrawidely tunable THz-wave sources (1-40 THz) [5]. The purpose of the presenting work is to devise a nondestructive measurement method for carrier density in semiconductors using a THz-wave source. GaN is one of the most remarkable semiconductors, as it has a wide bandgap, large heat capacity, and high breakdown voltage [6]. Here, we use our new measurement system to analyze a GaN substrate.

\section{THEORY}

To study the response of semiconductors to THz-waves, one may consider the dielectric function and contribution of optical phonons in the media applying the Drude model. The dielectric function $\varepsilon(\omega)$ for $\mathrm{THz}$-wave $\omega$ in semiconductors can be modeled as

$$
\varepsilon(\omega)=\varepsilon_{\infty}\left(1+\frac{\omega_{L}{ }^{2}-\omega_{T}{ }^{2}}{\omega_{T}{ }^{2}-\omega^{2}+i \omega \Gamma}-\frac{\omega_{p}(N)^{2}}{\omega(\omega-i \gamma)}\right),
$$

where $\omega_{L}$ and $\omega_{T}$ are the optical phonon frequencies of the longitudinal mode and transverse modes, respectively. The quantity, $\omega_{P}\left(=\sqrt{N e^{2} / \varepsilon_{0} m^{*}}\right)$ is a plasma frequency that is a function of carrier density $N$ in the medium, and $\Gamma$ and $\gamma$ are damping parameters for the optical phonons and carriers, respectively [7]. The relationship between reflectivity and dielectric function is

$$
R=\frac{\left(\varepsilon^{\prime}-1\right)^{2}+\varepsilon^{\prime \prime 2}}{\left(\varepsilon^{\prime}+1\right)^{2}+\varepsilon^{\prime \prime 2}}
$$

where $\varepsilon^{\prime}$ and $\varepsilon^{\prime \prime}$ represent the real and imaginary part of the dielectric function, respectively. Using (1) and (2), the reflectivity $R$ in semiconductors can be obtained.

When the THz-wave frequency is in the range from $\omega_{T}$ to $\omega_{L}$, the reflectivity $R_{\omega \sim \omega_{T}}$ is not dependent on the carrier density $N$ because the second term on the right side in (1), which does not include $N$, is dominant. 
This highly reflective band is well known as the reststrahlen band of ionic crystals. We used $\mathrm{THz}$-waves in this region as an intensity reference for the measurement of reflectivity. Furthermore, our results showed that the reflectivity $R_{\omega \neq \omega_{T}}$, which is out of the high reflective region, is useful for sensing the carrier density $N$. Frequency agility and wide tunability are required for a source that compares the THz-wave reflectivity between $\omega \sim \omega_{T}$ and $\omega \neq \omega_{T}$. The frequencies $\omega_{T}$ and $\omega_{L}$ in wurtzite GaN crystal were reported as $16.8 \mathrm{THz}$ and 22.3 $\mathrm{THz}$, respectively [8].

\section{EXPERIMENTAL SETUP}

The monochromatic ultra-widely tunable THz-wave source developed by our research group was used to measure the reflective spectra in GaN wafers. Figure 1 shows the experimental setup. A frequency-agile dual wavelength $\left(\lambda_{1}\right.$ and $\lambda_{2}$ ) source of around $1.3 \mu \mathrm{m}$ from KTP-OPO was used for collinear THz-difference frequency generation (DFG), satisfying the phase-matching condition in the DAST crystal [5]. This OPO consisted of two KTP crystals and two mirrors, which were pumped by the second harmonic of a Q-switched Nd:YAG laser $(532 \mathrm{~nm}, 8 \mathrm{~ns}$, and $100 \mathrm{~Hz}$ ). Here, M1 was coated for high reflectivity of the signal waves and high transmittance of $532 \mathrm{~nm}$ and idler waves (around $1.3 \mu \mathrm{m}$ ); M2 was a broadband silver mirror. In addition, M3 was coated for the high reflection of idler waves and high transmittance at $532 \mathrm{~nm}$. Each KTP crystal was mounted on a galvano scanner, and the angle was controlled independently. The KTPOPO was tunable within 1250 to $1700 \mathrm{~nm}$, enabling phasematched $\mathrm{THz}$-wave generation over a wide range. The idler waves from the KTP-OPO were focused onto a polished DAST crystal (Furukawa Co., Ltd.), and the THz wave was generated from the DAST crystal. The THz-wave frequency could change from pulse to pulse using synchronized control of the galvano scanner angle, with a pump-laser repetition frequency of $100 \mathrm{~Hz}$. A low-pass filter was placed in the wave path to block the $\lambda_{1}$ and $\lambda_{2}$ outputs. The THz wave, which was collimated using an off-axis parabolic mirror, was fed into a reflective imaging system. To make the reflective signal, an off-axis parabolic mirror and sample positions were controlled as shown in Figure 1. The movable distances of the sample stage and off-axis parabolic mirror were $85 \mathrm{~mm}$ in the $x$ and $y$ directions, respectively. The reflected $\mathrm{THz}$ wave was detected using a pyrodetector, which was operated at room temperature. The total $\mathrm{THz}$ range of 3 to $32 \mathrm{THz}$ of this reflective spectrometer is reduced by the tuning range of the KTP$\mathrm{OPO}$ and the sensitive range of a pyrodetector. In this experiment, the spatial resolution was estimated to be higher than 2 $\mathrm{mm}$, based on measurements of a line and space in a reflective image from a resolution target (Edmund Optics Inc., USAF Resolution Target 2" SQ Positive). The spatial resolution depends on the spot size of pump beams in a DAST crystal, and the focusing lengths of a pair of off-axis parabolic mirrors for the collimation and focusing of the THz-wave onto a sample. We could achieve a maximum resolution of $0.5 \mathrm{~mm}$ changing these factors relative to the $\mathrm{THz}$-wave power and depth of field (DOF) of the reflective image.

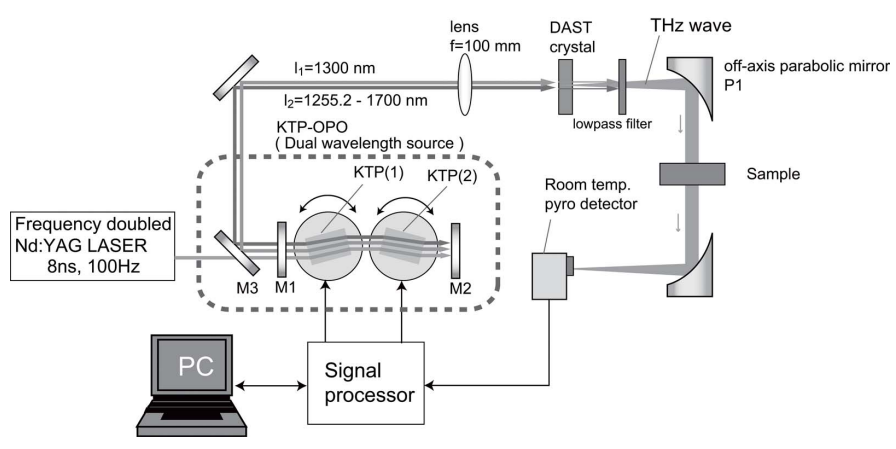

FIG. 1 Experimental setup for THz-reflective imaging measurement.

\section{RESULTS AND DISCUSSION}

We prepared three samples of n-type GaN substrates with different carrier densities. These n-type GaN samples were made by doping silicon. The carrier density of silicon is changed by varying the volume of $\mathrm{SiH}_{2} \mathrm{Cl}_{2}$ (dichlorosilane). This dichlorosilane flows when the $\mathrm{GaN}$ wafer is grown on a sapphire substrate via hydride vapor phase epitaxy (HVPE). The carrier density of the GaN substrates, which were measured by secondary ion-microprobe mass spectrometer (SIMS), were 1) $2.5 \times 10^{16} \mathrm{~cm}^{-3}$, 2) $1.0 \times 10^{18} \mathrm{~cm}^{-3}$, and 3) $1.5 \times 10^{18} \mathrm{~cm}^{-3}$.

The reflective spectra shown in Figure 2 were measured at the center of the GaN substrates. In the frequency region above $10 \mathrm{THz}$, they were in agreement with (2) for each carrier density, with values of $\Gamma=17 \mathrm{~cm}^{-1}, \gamma=12 \mathrm{~cm}^{-1}$, $\omega_{L}=744 \mathrm{~cm}^{-1}, \omega_{T}=560 \mathrm{~cm}^{-1}, m^{*}=0.2 \mathrm{~m}$, and $\varepsilon_{\text {inf }}=5.35$ used $[6,9] . \gamma$ was obtained from the typical value of the mobility $\mu=150 \mathrm{~cm} /$ Vs from the literature. Note that the larger $\gamma\left(>100 \mathrm{~cm}^{-1}\right)$ was necessary for agreement in a wider frequency region.

We mapped $\mathrm{THz}-$-reflective intensities for all samples aligned in the region of $18 \mathrm{~mm} \times 40 \mathrm{~mm}$ with frequencies switched to $19.2,22.7$, and $26.5 \mathrm{THz}$ with averaging in each set of 160 pulses. These values were selected because of their good signal-to-noise ratios within the reference and sensing regions. We used the 19.2-THz map as a reference for reflective intensity because the carrier density had no effect on the reflectivity at this frequency, as shown in Figure 2.

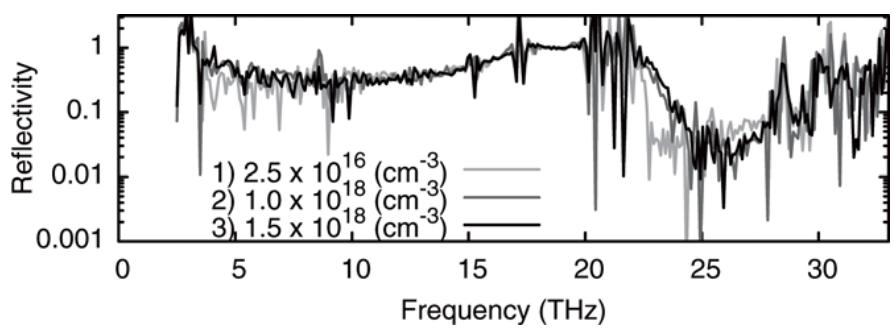

FIG. 2 THz reflective spectrum in GaN substrates with carrier density of 1 ) $2.5 \times 10^{16} \mathrm{~cm}^{-3}$, 2) $1.0 \times 10^{18} \mathrm{~cm}^{-3}$, and 3) $1.5 \times 10^{18} \mathrm{~cm}^{-3}$.

The normalized reflective images at 22.7 and $26.5 \mathrm{THz}$ for each sample are shown in Figure 3. The reflective intensities at 22.7 or $26.5 \mathrm{THz}$ differed among the samples, and the reflectivity depended on the carrier density. Figure 4 shows the relationships between carrier density and relative reflectivity averaged over all pixels for samples at 22.7 and $26.5 \mathrm{THz}$. The rel- 
ative reflectivity at 22.7 (26.5) $\mathrm{THz}$ increased (decreased) with the carrier density. In this Figure 4, broken and solid curves are respectively calculated with the values from the literature as mentioned above, and the values $\Gamma=50 \mathrm{~cm}^{-1}, \gamma=200 \mathrm{~cm}^{-1}$, and $\omega_{L}=750 \mathrm{~cm}^{-1}$, which were chosen to fit the curves to the experimental results. Although further quantitative studies are necessary, theoretical analyses and experimental results showed similar behavior to that found here.
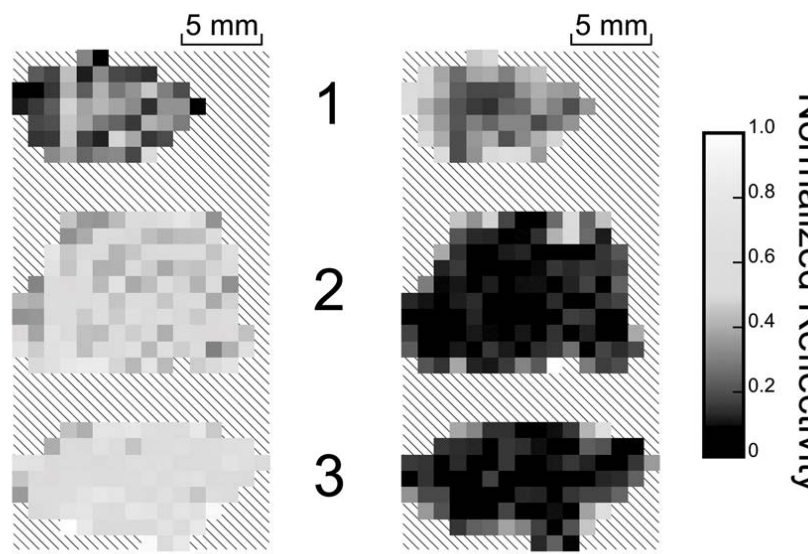

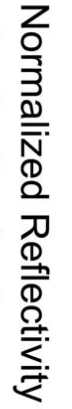

$\begin{array}{ll}\text { (a) } R(22.7 \mathrm{THz}) / R(19.2 \mathrm{THz}) & \text { (b) } R(26.5 \mathrm{THz}) / R(19.2 \mathrm{THz})\end{array}$

FIC. 3 Reflective images in three GaN substrates. Panels (a) and (b) respectively depict reflective images for $22.7 \mathrm{THz}$, and $26.5 \mathrm{THz}$ normalized by the reflective intensity at 19.2 THz . The sample numbers 1,2 , and 3 respectively denote GaN substrates with carrier densities of $2.5 \times 10^{16} \mathrm{~cm}^{-3}, 1.0 \times 10^{18} \mathrm{~cm}^{-3}$, and $1.5 \times 10^{18} \mathrm{~cm}^{-3}$. The hatched area indicates the outside of the samples.

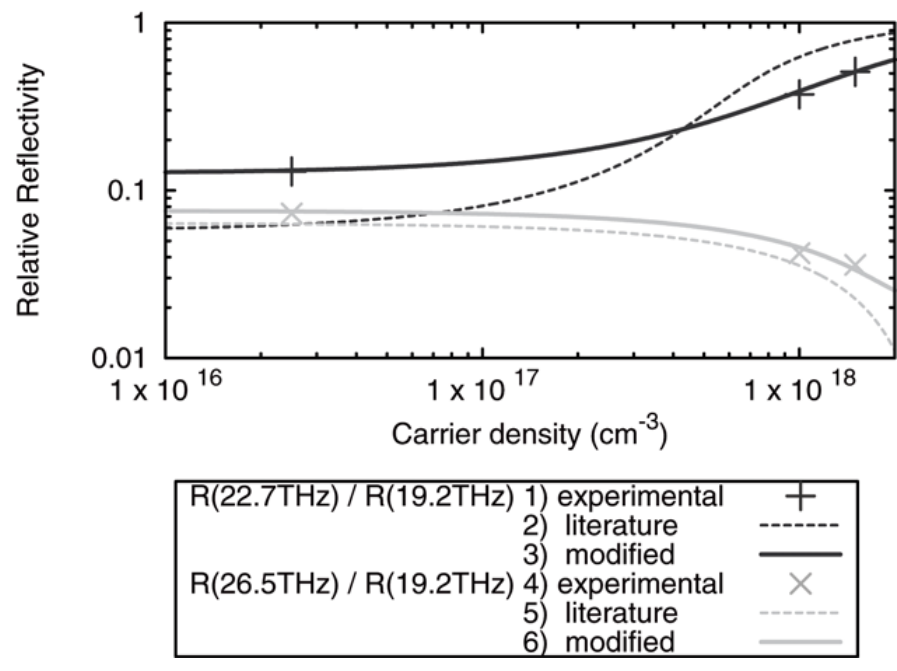

FIG. 4 Carrier density vs. relative reflectivity of 1), 2), 3) $22.7 \mathrm{THz}$ and 4), 5), 6) $26.5 \mathrm{THz}$ for $19.2 \mathrm{THz}$. 1), 4) are experimental results. Broken and solid curves are theoretical analysis using the values in literatures 2), 5) $[6,9]$ and the modified values 3$), 6$ ), respectively.

We found that the relative density resolution $\Delta N$ was defined by the fluctuation of measured reflectivity, which mainly depended on the power fluctuation of the $\mathrm{THz}$ wave in each pulse, its averaging number, and the internal noise of the pyrodetector. The relative accuracy of carrier density $\frac{\Delta N}{N}=\frac{\Delta R}{N\left(\frac{d R}{d N}\right)}$ for a sample of $\mathrm{N}=1 \times 10^{18} \mathrm{~cm}^{-3}$ was estimated to be approximately 0.2 using (2) and the fluctuation of measured reflectivity $\Delta R<5 \%$ at $22.7 \mathrm{THz}$ and averaging the number 1000 pulses per pixel in the current system.

\section{CONCLUSION}

We developed a nondestructive method for measuring the carrier density distribution on a surface of semiconductors based on a THz-reflective mapping system. We observed differences in the carrier density in a GaN wafer, and the reflectivity behavior was in agreement with the theoretical analysis. A quantitative analysis to convert reflectivity to carrier density by a numerical fitting method is the next step in this study. We demonstrated an extremely useful application of THz waves: a THz-reflective imaging system for carrier density measurement in semiconductors.

\section{References}

[1] N. Harrick, "Semiconductor surface properties deduced from free carrier absorption and reflection of infrared radiation" J. Phys. Chem. Solids 14, 60 (1960).

[2] J. Majewski and D. Matthiesen, "Quantative infrared imaging for measurement of dopant distribution in gallium arsenide" J. Cryst. Growth 137, 249 (1994).

[3] C. Drake, S. Deshpande, and S. Seal, "Determination of free carrier density and space charge layer variation in nanocrystalline $\mathrm{In}^{3+}$ doped tin oxides using Fourier transform infrared spectroscopy" Appl. Phys. Lett. 89, 143116 (2006).

[4] A. J. Huber, F. Keilmann, J. Wittborn, J. Aizpurua, and R. Hillenbrand, "Terahertz Near-Field Nanoscopy of Mobile Carriers in Single Semiconductor Nanodevices" Nano Lett. 8, 3766-3770 (2008).

[5] H. Ito, K. Suizu, T. Yamashita, A. Nawahara, and T. Sato, "Random Frequency Accessible Broad Tunable Terahertz-Wave Source Using Phase-Matched 4-Dimethylamino- $\mathrm{N}$-methyl-4-stilbazolium Tosylate Crystal" Jpn. J. Appl. Phys. 46, 7321 (2007).

[6] V. Bougrov, M. E. Levinshtein, S. L. Rumyantsev, and A. Zubrilov, Properties of Advanced Semiconductor Materials GaN, AIN, InN, BN, SiC, SiGe (John Wiley at Sons, Inc., New York, 2001).

[7] R. Holm, J. Gibson, and E. Palik, "Infrared reflectance studies of bulk and epitaxial-film n-type GaAs" J. Appl. Phys. 48, 212 (1977).

[8] A. Barker and M. Ilegems, "Infrared Lattice Vibrations and FreeElectron Dispersion in GaN" Phys. Rev. B 7, 743 (1973).

[9] Y. Fu, M. Willander, Z.-F. Li, and W. Lu, “Electron mobilities, Hall factors, and scattering processes of n-type GaN epilayers studied by infrared reflection and Hall measurements" Phys. Rev. B 67, 113313 (2003). 